\title{
Identification of novel diagnostic biomarkers for thyroid carcinoma
}

\author{
Xiliang Wang ${ }^{1,2}$, Qing Zhang ${ }^{1}$, Zhiming Cai ${ }^{1}$, Yifan Dai ${ }^{3}$ and Lisha Mou ${ }^{1}$ \\ ${ }^{1}$ Shenzhen Xenotransplantation Medical Engineering Research and Development Center, Institute of Translational Medicine, \\ Shenzhen Second People's Hospital, First Affiliated Hospital of Shenzhen University, Shenzhen 518035, China \\ ${ }^{2}$ Department of Biochemistry in Zhongshan School of Medicine, Sun Yat-Sen University, Guangzhou 510080, China \\ 3 Jiangsu Key Laboratory of Xenotransplantation, Nanjing Medical University, Nanjing 210029, China \\ Correspondence to: Lisha Mou, email: lishamou@gmail.com \\ Keywords: thyroid carcinoma; bioinformatics; dysregulation network; biomarker \\ Received: June 27, $2017 \quad$ Accepted: November 19, $2017 \quad$ Published: December 04, 2017 \\ Copyright: Wang et al. This is an open-access article distributed under the terms of the Creative Commons Attribution License 3.0 \\ (CC BY 3.0), which permits unrestricted use, distribution, and reproduction in any medium, provided the original author and source \\ are credited.
}

\section{ABSTRACT}

Thyroid carcinoma (THCA) is the most universal endocrine malignancy worldwide. Unfortunately, a limited number of large-scale analyses have been performed to identify biomarkers for THCA. Here, we conducted a meta-analysis using 505 THCA patients and 59 normal controls from The Cancer Genome Atlas. After identifying differentially expressed long non-coding RNA (IncRNA) and protein coding genes (PCG), we found vast difference in various IncRNA-PCG co-expressed pairs in THCA. A dysregulation network with scale-free topology was constructed. Four molecules (LA16c-380H5.2, RP11-203J24.8, MLF1 and SDC4) could potentially serve as diagnostic biomarkers of THCA with high sensitivity and specificity. We further represent a diagnostic panel with expression cutoff values. Our results demonstrate the potential application of those four molecules as novel independent biomarkers for THCA diagnosis.

\section{INTRODUCTION}

Thyroid carcinoma (THCA) is the most widespread endocrine malignancy worldwide, with an incidence rate that increases by $4 \%$ every year $[1,2]$. Tissue biopsy is the current gold standard for diagnostic tests for cancers; however, biopsy results are often inevitably subjective because of differing reporting methods among pathologists or limited diagnostic accuracy associated with sampling error [3]. For THCA, indeterminate (10-20\%) and inadequate (10-15\%) conclusions perplex clinicians and undermine the diagnostic value of biopsy procedures [4], and the use of core needle biopsy for the thyroid is currently limited by a lack of well-accepted diagnostic criteria [5]. Some THCAs are associated with aggressive clinical behavior or a poor prognosis [6]. Therefore, finding more sensitive and specific biomarkers to use for the early detection of THCA is undoubtedly of great significance. However, there is still no routine application of these markers in clinical practice. Progress in understanding the molecular basis of cancers using omics technologies has provided opportunities to develop novel tools to diagnose, predict cancers and evaluate treatment responses [7].

Long noncoding RNA (lncRNA) is unable to be translated into proteins, with transcript lengths of more than 200 nucleotides $[8,9]$. LncRNA has emerged as a vital regulator in biological, developmental and pathological processes of tissues and diseases including various cancers through mechanisms such as chromatin reprogramming, cis regulation at enhancers and posttranscriptional regulation of mRNA processing $[10,11]$. Cancer-related IncRNAs showed aberrant expression patterns in tissue- or cancer type-specific manners, suggesting their potentials as novel independent and promising biomarkers for cancer diagnosis or prognosis [12-16]. Recently, a very large-scale data analysis found 1289 THCA-associated lncRNAs, just after renal clear cell carcinoma (1429 associated lncRNAs), in 27 types of cancers [10]. However, a few lncRNAs were declared to be implicated in the development and progression of THCA. For instance, IncRNAs BANCR and PVT1 were 
overexpressed, and NAMA and PTCSC3 were downregulated in THCA patients [17-20]. To date, limited knowledge is known about the diagnostic or prognostic values of IncRNA in THCA [21]. In this study, next generation sequencing (NGS) datasets from The Cancer Genome Atlas (TCGA) [22] were used to identify two lncRNAs (LA16c-380H5.2 and RP11-203J24.8) and two protein coding genes (PCGs) (MLF1 and SDC4) as potential diagnostic biomarkers with high sensitivity and specificity.

\section{RESULTS}

\section{Transcriptome expression profiles in THCA and normal samples}

We compared expression levels of PCG and lncRNA between 505 cancer and 59 normal samples (sample list in Supplementary Table 1). LncRNA was expressed at evidently lower levels than PCG in both cancer and normal samples ( $\mathrm{P}<1 \mathrm{E}-100)$, showing a low abundance of lncRNA in cells (Figure 1A and 1B), which is consistent with previous studies [23, 24]. When compared with normal samples, the expression perturbation observed for
lncRNA was more significant in THCA $(\mathrm{P}=0.02$, Figure $1 C)$, while $P C G$ variance was considerably weaker in cancer samples $(P=0.32$, Figure $1 D)$, partly because that the specific expression of lncRNA is a vital regulator in gene expression.

To identify differentially expressed PCGs and lncRNAs in THCA and normal thyroid glands, we applied a binary statistical analysis based on the number of zero-values in the expression level (read count $=0$ ). We identified 690 up-regulated and five down-regulated PCGs, and 1634 up-regulated and 21 down-regulated lncRNAs with $\mid$ fold change (FC) $\mid>2$ and FDR $<0.01$ by Fisher's exact test; as well as 1273 up-regulated and 880 down-regulated PCGs, and 341 up-regulated and 591 down-regulated lncRNAs with $|\mathrm{FC}|>2$ and FDR $<0.05$ by Student's t test (Figure 1E). In total, we found that 2848 PCGs and 2587 lncRNAs were differentially expressed between THCA and normal tissue (Supplementary Table 2). Among them, 1963 PCGs and 1975 lncRNAs were up-regulated, while 885 PCGs and 612 lncRNAs were down-regulated (Figure 1F). Up-regulated features were found to be much more common than down-regulated features ( $>2$ times), which is similar to the expression patterns in two other reports about THCA $[4,25]$; these
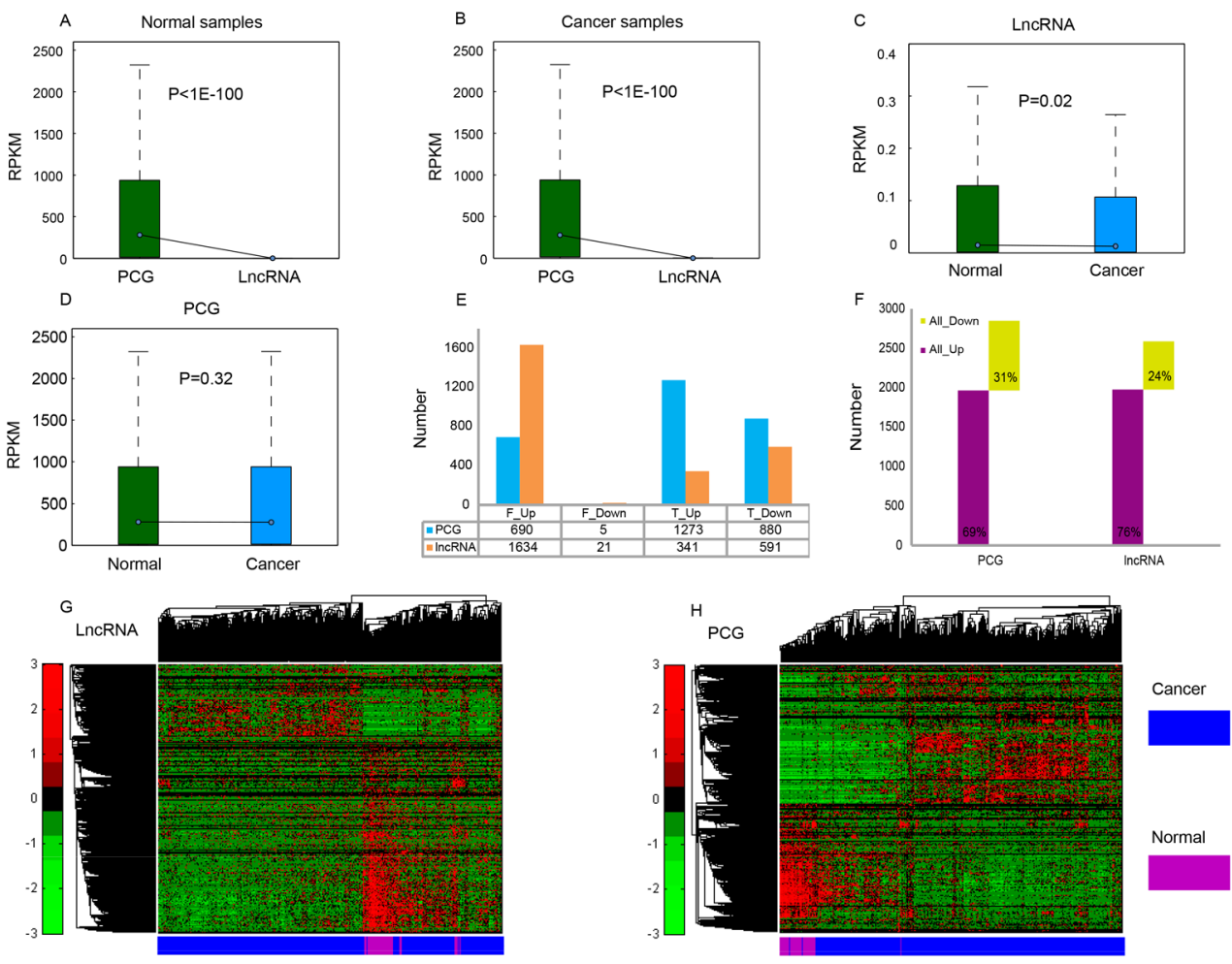

Figure 1: Expression profiles of PCG and IncRNA in cancer and normal samples. Average expression levels (RPKM) of PCG and lncRNA in normal samples (A) and THCA samples (B). RPKM comparison of lncRNA (C) and PCG (D) between normal samples and cancer samples. (E) The number of up- and down-regulated PCG and lncRNA identified through Fisher's exact test and Student's t test, respectively. F_Up/F_Down: up- or down-regulated PCG or lncRNA identified through Fisher's exact test, T_Up/T_Down: up- or downregulated PCG or lncRNA identified through Student's t test. (F) Total number of differentially expressed PCG and lncRNA. Unsupervised hierarchical clustering using expression profiles of $\operatorname{lncRNA}(\mathbf{G})$ and PCG $(\mathbf{H})$ revealed distinct separation of cancer samples from normal samples. 
Table 1: Number of differentially expressed IncRNA-PCG co-expression pairs

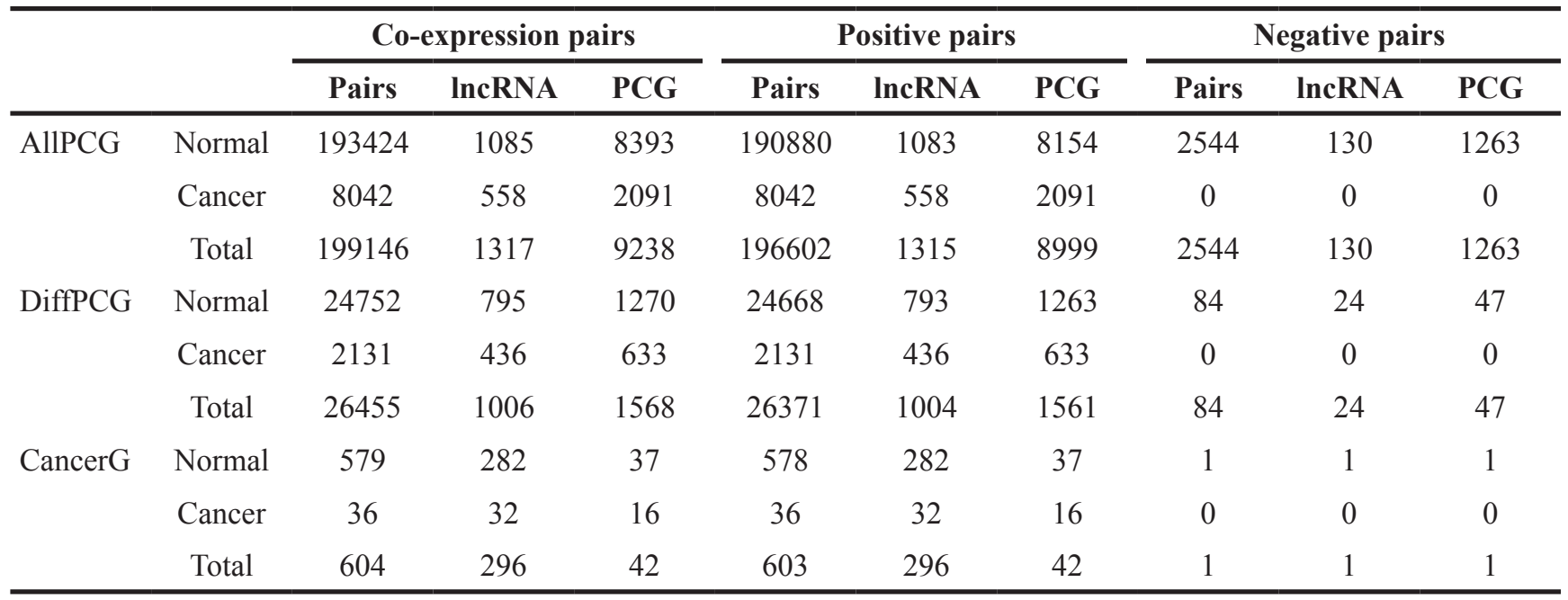

Co-expressed pairs were defined with cutoff of $|\mathrm{PCC}| \geq 0.7$ and $\mathrm{P}<0.001$.

3th-5th column: number of co-expressed pairs between PCGs and differentially expressed lncRNAs.

6th-8th column: number of positively co-expressed pairs between PCGs and differentially expressed lncRNAs.

9th-11th column: number of negatively co-expressed pairs between PCGs and differentially expressed lncRNAs.

3th-4th row: number of co-expressed pairs between all PCGs in the Ensembl reference ("AllPCG" for short) and

differentially expressed lncRNAs in normal (3th row) or THCA samples (4th row).

6th-7th row: number of co-expressed pairs between differentially expressed PCGs ("DiffPCG" for short) and differentially

expressed lncRNAs in normal (6th row) or THCA samples (7th row).

9th-10th row: number of co-expressed pairs between those differentially expressed PCGs that were also cancer genes

("CancerG" for short) and differentially expressed lncRNAs in normal (9th row) or THCA samples (10th row).

results likely indicate that the demands of cancer cells for quick proliferation, tissue invasion and metastasis are met. A hierarchical cluster analysis of differentially expressed lncRNA (Figure 1G) and PCG (Figure 1H) showed that THCA patients were well discriminated from normal individuals according to their expression levels, indicating the possibility of selecting a group of features for THCA diagnosis.

Pathway analysis of differentially expressed PCGs indicated that 34 pathways were enriched significantly ( $\mathrm{P}$ $<0.05$, Supplementary Table 3). Many of these pathways were linked to cancer, such as "pathways in cancer" (P $=0.028)$, "cytokine-cytokine receptor interaction" $(\mathrm{P}=$ 8.54E-6), which was in accordance with an early research [2], "PPAR signaling pathway" $(\mathrm{P}=0.022)$, which was particularly associated with THCA [26, 27], and "PI3KAkt signaling pathway" $(\mathrm{P}=1.63 \mathrm{E}-4)$, which was one of the most important molecular mechanisms identified in the carcinogenesis of THCA [28].

\section{Dysregulated network of differentially expressed features}

To explore the dysregulation of differentially expressed lncRNAs involved in THCA tumorigenesis and development, we calculated Pearson's correlation coefficient (PCC) by examining the paired lncRNA and PCG expression profiles. In normal samples, 1085 lncRNAs were identified to have 8393 target PCGs with 193424 co-expressed pairs using all PCGs in the Ensembl reference ("AllPCG" for short). Among them, 1083 IncRNAs were positively correlated with 8154 PCGs with 190880 co-expressed pairs, while only 130 IncRNAs were negatively correlated with 1260 PCGs with 2544 co-expressed pairs (Table 1). When using those differentially expressed PCGs ("DiffPCG" for short), 795 lncRNAs were found to have 1270 target PCGs with 24752 co-expressed pairs. Among those, 793 lncRNAs were positively correlated with 1263 PCGs with 24668 pairs, while only 24 lncRNAs were negatively correlated with 47 PCGs with 84 pairs (Table 1). As for those differentially expressed PCGs that were also cancer genes (cancer genes were cited from the report of Mathias Uhlén et al. [29]; "CancerG" for short, Supplementary Figure 1), 282 lncRNAs had 37 target PCGs with 579 co-expressed pairs. All of the $282 \operatorname{lncRNAs}$ were positively correlated with 37 PCGs with 578 pairs, while just one lncRNA was negatively correlated with one PCG with one pair (Table 1). Although there were positive and negative coexpression pairs of lncRNA-PCG in normal samples, the former was far more common than the latter.

In THCA patients, 558 lncRNAs had 2091 targets with 8042 co-expressed pairs in the "AllPCG" level. 436 lncRNAs had 633 targets with 2131 co-expressed pairs in 
Table 2: Four types of dysregulated pairs among co-expressed pairs of IncRNA-PCG

\begin{tabular}{|c|c|c|c|c|c|c|c|c|c|c|c|}
\hline \multirow{2}{*}{ Type } & \multirow{2}{*}{ Normal } & \multirow{2}{*}{ Cancer } & \multicolumn{3}{|c|}{ AllPCG } & \multicolumn{3}{|c|}{ DiffPCG } & \multicolumn{3}{|c|}{ CancerG } \\
\hline & & & IncRNA & PCG & Pairs & IncRNA & PCG & Pairs & IncRNA & PCG & Pairs \\
\hline I & Yes & No & 1060 & 8344 & 191104 & 764 & 1237 & 24324 & 281 & 36 & 568 \\
\hline II & No & Yes & 490 & 1634 & 5722 & 371 & 549 & 1703 & 23 & 14 & 25 \\
\hline III & Positive & Negative & 0 & 0 & 0 & 0 & 0 & 0 & 0 & 0 & 0 \\
\hline IV & Negative & Positive & 0 & 0 & 0 & 0 & 0 & 0 & 0 & 0 & 0 \\
\hline Total & & & 1298 & 9202 & 196826 & 985 & 1546 & 26027 & 295 & 42 & 593 \\
\hline
\end{tabular}

I: co-expressed pairs appeared in normal samples, while disappeared in THCA. II: co-expressed pairs disappeared in normal samples, while appeared in THCA. III: pairs positively co-expressed in normal samples, while negatively co-expressed in THCA. IV: pairs negatively co-expressed in normal samples, while positively co-expressed in THCA.

AllPCG: all protein coding genes (PCGs) in the Ensembl reference. DiffPCG: differentially expressed PCGs. CancerG: differentially expressed PCGs that were also cancer genes.

The number of lncRNA in this table is the number of differentially expressed lncRNAs between normal and THCA samples.

the "DiffPCG" level. When using "CancerG", 32 lncRNAs had 16 targets with 36 co-expressed pairs (Table 1). It was astounding that all of these lncRNAs were positively correlated with PCGs in THCA samples without negative regulation, showing that PCGs followed the same trends as lncRNAs (Figure 1F). Moreover, the number of coexpressed pairs in THCA patients was significantly less than in normal samples, only approximately $4.16 \%(8042 / 193424)$ in the "AllPCG" level, $8.61 \%$ $(2131 / 24752)$ in the "DiffPCG" level, and $6.22 \%$ $(36 / 579)$ in the "CancerG" level (Table 1), illustrating a massive loss in regulation of lncRNAs to PCGs in THCA tumorigenesis or development. This agreed with the idea that most characterized lncRNAs display deregulated expression in cancers, suggesting they may play oncogenic or tumor suppressive functions [30].

Four types of dysregulated pairs among lncRNAPCG co-expressed pairs are defined in Table 2. There were 196826, 26027, and 593 dysregulated pairs, accounting for $98.84 \%$ (out of 199146), 98.38\% (out of 26455), and 98.18\% (out of 604) in "AllPCG", "DiffPCG", and "CancerG" levels, respectively (Table 1 and Table 2). In particular, in the "CancerG" level, nearly $100 \%$ of the lncRNAs (295/296) and PCGs (42/42) showed dysregulation (Table 2). It is no surprise that the overwhelming majority of the population was Type I; there were no Type III and Type IV dysregulated pairs (Table 2). Those lost (Type I) and gained (Type II) dysregulated pairs may be one important reason for the aberrance of THCA. These results showed dramatic turbulence in the regulation roles of IncRNA to PCG in THCA, supporting the findings that lncRNAs are frequently dysregulated in various tumors [31-33].

Both in Table 1 and Table 2, there was an intriguing phenomenon where the number of lncRNAs was much lower than that of PCG in both "AllPCG" and "DiffPCG" levels, which was concordant with the idea that lncRNA could target multiple, even hundreds of genes in the human genome $[34,35]$. This is just the opposite in the "CancerG" level where one PCG was influenced by several lncRNAs, suggesting key roles of those 42 cancer PCGs in THCA (42 PCGs are listed in Supplementary Table 4). For example, ENSG00000105976 (c-Met) is an oncogene protein with tyrosine kinase activity, and its abnormal activation has been detected in various cancers, including THCA [36, 37]. Another example is that ENSG00000066468 (FGFR-2), which has been implicated in the onset of THCA, was reduced in THCA $[38,39]$. In agreement with the above reports, c-Met was aberrantly up-regulated $(\mathrm{P}<2.2 \mathrm{E}-16, \mathrm{FC}=5.99)$, and FGFR-2 was aberrantly down-regulated $(\mathrm{P}<2.2 \mathrm{E}-16, \mathrm{FC}=2.3)$ in our study (Supplementary Figure 2A and 2B). Pathway analysis of those 42 PCGs showed that "Transcriptional misregulation in cancer" was the most enriched pathway $(\mathrm{P}=1.11 \mathrm{E}-7)$, followed by "Pathways in cancer" $(\mathrm{P}=$ 2.83E-3), "PI3K-Akt signaling pathway" $(\mathrm{P}=8.32 \mathrm{E}-$ $3)$, "Cytokine-cytokine receptor interaction" $(\mathrm{P}=0.01)$, "Acute myeloid leukemia" $(\mathrm{P}=0.019)$, and "Central carbon metabolism in cancer" ( $\mathrm{P}=0.024)$ (Figure 2A). All of those six pathways are associated with cancer, these key pathways are regulated by lncRNA in THCA.

Therefore, we focused on the co-expressed network based on dysregulation pairs of those 42 PCGs and 295 regulating lncRNAs (Table 2). There were 337 (42 and 295) nodes and 593 edges in the network (Figure 2B), which had a scale-free topology with degree distribution following a power law (Figure 2C). Such a scale-free network has been found in many different organizational levels, ranging from genetics to protein interaction and protein domains [40, 41]. A distinguishing feature of such a scale-free network is the existence of a few highly connected nodes [42]. There were $117(72.7 \%)$ nodes of 
a degree lower than three, while 23 (6.8\%) nodes had degrees of six or above. To test the existence of separable functional units, we tried to mine feasible sub-modules. Two sub-networks were finally found (Figure 2D and 2E). There were 26 (Figure 2D) and 19 (Figure 2E) lncRNAs and only five of the same PCGs in those two sub-modules. A uniquely enriched pathway of all five PCGs (FSTL3, KLF6, MLF1, NR4A3, and SDC4) was "Transcriptional misregulation in cancer" $(\mathrm{P}=0.048)$, suggesting that those five PCGs and their regulating lncRNAs may play roles in transcriptional regulation levels in THCA. It also suggests their potential as biomarkers or therapeutic targets in THCA.

\section{Diagnostic values of LA16c-380H5.2, RP11- 203J24.8, MLF1 and SDC4}

The 505 patients were classified into either a highrisk group or a low-risk group by sub-module $1(\mathrm{P}=0.028$, Figure 3A), instead of sub-module $2(\mathrm{P}=0.093$, Figure $3 \mathrm{~B})$, demonstrating a significant difference in estimated survival time. Just four of the 31 elements in sub-module 1 showed predictive power, and were able to independently distinguish low-risk patients from high-risk individuals at a statistically significant level of 0.1 . Those four elements were LA16c-380H5.2 (Figure 3C), RP11-203J24.8 (Figure 3E), MLF1 (Figure 3G), and SDC4 (Figure 3I); the other 27 elements are shown in Supplementary Figure 3. Patients in the high-risk group had a lower survival ratio (Figure 3C, 3E, 3G, 3I) and shorter survival time (Supplementary Table 5) than those in the low-risk group. ROC (receiver operating characteristic) curves were applied to evaluate whether those four elements could distinguish high- or low-risk status. Surprisingly, the AUC (area under the ROC curve) was one with RPKM (reads per kilobase per million mapped) cutoff values of $0.346,0.327,311.258$, and 18095.531 for LA16c-380H5.2, RP11-203J24.8, MLF1, and SDC4, respectively (Supplementary Figure 4). These RPKM cutoff values could completely differentiate high- or low-risk status with P values less than 1.0E-10 and FC values of more than two (Figure 3D, 3F, 3H and 3J). The expression of LA16c-380H5.2 and SDC4 tended to be up-regulated, while the remaining two (RP11-203J24.8 and MLF1) were down-regulated for patients in the highrisk group, suggesting that their expression levels were closely related to the development of THCA. Interestingly, they all showed obviously different expressions levels in the low risk and normal groups (Figure 3D, 3F, 3H and 3J).

ROC curves were further generated to evaluate whether they could diagnose THCA. For LA16c-380H5.2, the AUC reached 0.781 (95\% CI: 0.720-0.843) when using the RPKM cutoff value of 0.151 , and the sensitivity and specificity were 0.677 and 0.847 , respectively (Figure 4A). For RP11-203J24.8, these values are an AUC of 0.871 (95\% CI: 0.825-0.917), a RPKM cutoff value of 0.195 , and a sensitivity and specificity of 0.838 and 0.780 (Figure 4B). For MLF1, the AUC was 0.924 (95\% CI: $0.886-0.962)$, the RPKM cutoff value was 310.115 , and the sensitivity and specificity were 0.863 and 0.864 (Figure 4C). For SDC4, the AUC was 0.898 (95\% CI: $0.870-0.926)$, the RPKM cutoff value was 4769.11 , and the sensitivity and specificity were 0.838 and 0.881 (Figure
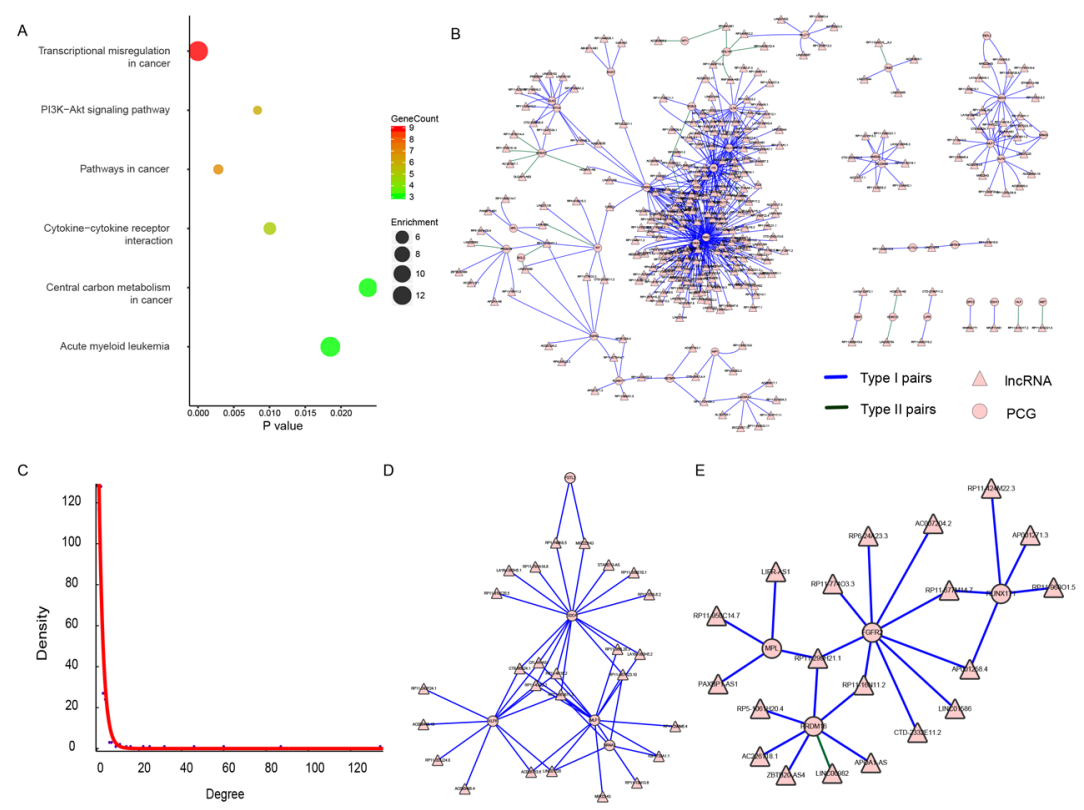

Figure 2: The IncRNA-PCG dysregulation network of the 42 PCGs. (A) Results of KEGG pathway enrichment, showing P value, gene count, and fold enrichment in each pathway. (B) The dysregulation network of the 42 PCGs. The lncRNA was indicated to triangle and PCG was indicated to circle. The color of blue represents Type I (disappearance) pairs and green represents Type II (appearance) pairs. (C) The exponential distribution of degree of each node in (B). (D) and (E) were two sub-modules identified from (B). 
4D). THCA can be diagnosed accurately using expression levels of these four elements (Figure 4E-4H). These analyses showed that these four elements might serve as outstanding diagnostic biomarkers for THCA, which would be useful in screening biopsies in a histopathologic setting. We confirmed the expression pattern of the SDC4 by other samples (GSE3467 and GSE3678 [43]) from GEO datasets (Figure 5A and Supplementary Figure 5A). And, we also had experimental data about the expression of the remaining three genes between THCA cell lines (TT, B-CPAP and BHT101) and normal thyroid cell lines (HT-ori3) (Figure 5B-5D and Supplementary Figure 5B and $5 \mathrm{C}$ ).

As shown in (Figure 4E-4H) and (Figure 3D, 3F, $3 \mathrm{H}, 3 \mathrm{~J}), \mathrm{LA} 16 \mathrm{c}-380 \mathrm{H} 5.2$ and SDC4 were up-regulated in THCA patients compared to normal samples and also tended to be expressed at higher levels in the high-risk group compared to the low-risk group. In contrast, MLF1 was down-regulated in THCA patients when compared with normal samples and also tended to be expressed at a lower level in the high-risk group compare with the low-risk group. RP11-203J24.8 was also differentially expressed among the normal sample, the low-risk group and the high-risk group. These expression signatures are shown in (Figure 4I), suggesting that these four elements could diagnose THCA patients from normal individuals.
Based on the cutoff values of RPKM, they may be divided into two groups with LA16c-380H5.2 and SDC4 in Group I and MLF1 and RP11-203J24.8 in Group II (Figure 4J). Group I could explicitly differentiate the normal, low-risk and high-risk groups. For LA16c-380H5.2, an individual would be diagnosed as normal with an RPKM value less than 0.151 , as high-risk with an RPKM value higher than 0.346, and as low-risk with an RPKM value between 0.151 and 0.346 . For SDC4, an individual would be considered healthy with an RPKM value less than 4769.11, as highrisk with an RPKM value more than 18095.531, and as low-risk with an RPKM value between 4769.11 and 18095.531. The results of Group II seemed ambiguous, but they may be an accessory to Group I. MLF1, SDC4, LA16c-380H5.2, and RP11-203J24.8 were not in the list of pan-cancer (including THCA) biomarkers reported by Bogumil Kaczkowski et al. [44]. Likewise, they were not expressed differentially in a pan-cancer (not including THCA) transcriptome analysis published by Christopher $R$ Cabanski et al. [45]. This means that those expression signatures (Figure 4J) could be used to develop a novel diagnostic panel for THCA patients. In comparison, PAX8 (paired box 8), which encodes a member of the paired box family of transcription factors, was involved in thyroid follicular cell development and was thyroid-specifically expressed [46]. TTF1 (thyroid transcription factor 1) was
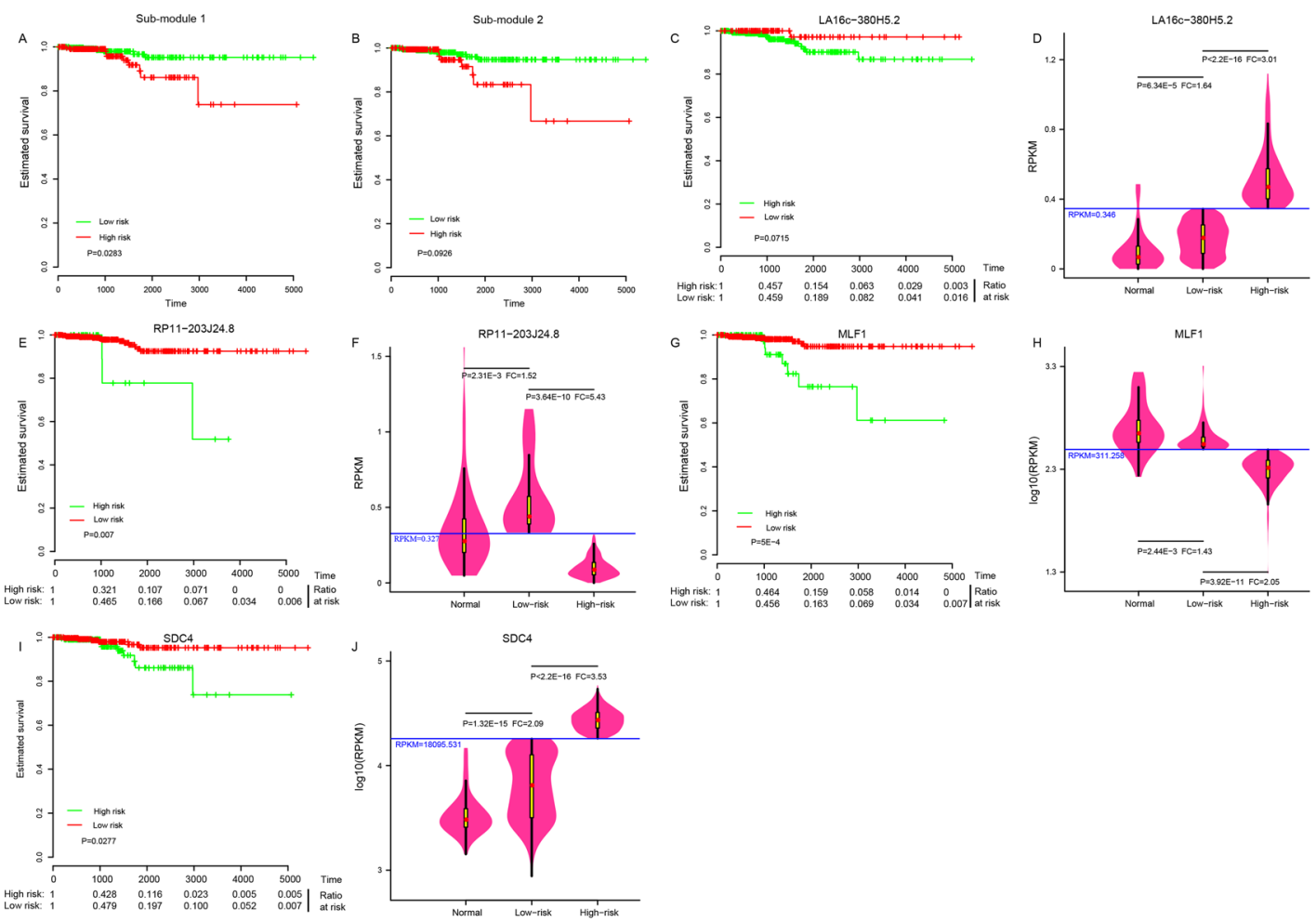

Figure 3: Four potential biomarkers. Sub-module 1 (A), instead of sub-module 2 (B) could distinguish the high-risk group from the low-risk group in THCA patients. Four elements in sub-module 1: LA16c-380H5.2 (C), RP11-203J24.8 (E), MLF1 (G) and SDC4 (I) could distinguish the high-risk group from the low-risk group. Comparison of expression levels in the normal, the low-risk and the high-risk groups for LA16c-380H5.2 (D), RP11-203J24.8 (F), MLF1 (H) and SDC4 (J). 
considered to be a thyroid transcription factor that encoded one homeobox protein [47]. They were identified as two potential biomarkers of THCA [48]. In the present study, both PAX8 and TTF1 were expressed at lower levels in THCA patients than in normal samples; however, they were not notable enough to be biomarker candidates, with FC values less than two (Supplementary Figure 2C and 2D).

To further examine whether these four biomarkers were THCA-specific or thyroid-specific, we retrieved their expression levels from three other independent datasets (Genecards [49], cBioPortal [50], and NONCODE [51]) in FPKM (fragments per kilobase per millions) values. LA16c-380H5.2, which was not found in NONCODE, was found at a low expression level (mean FPKM = 0.16) in the thyroid (Figure 6A). RP11-203J24.8 matched no results in Genecards and cBioPortal, but was expressed in low levels (mean FPKM = 0.365) in the thyroid (Figure 6B). MLF1 was expressed moderately (mean FPKM $=14.44$ ) in the thyroid (Figure 6C). SDC4 was highly expressed (mean FPKM = 75.69) in the thyroid (Figure 6D). All four biomarkers were pervasively expressed in most major human tissues and did not show a thyroidspecific expression pattern. MLF1 was moderately expressed (mean FPKM $=233.73$, Figure 6E), while
SDC4 was apparently highly expressed (mean FPKM = 19772.64, Figure 6F) in THCA, among various cancers. Although MLF1 and SDC4 were generally highly expressed in multiple types of cancers, their expression levels varied over a large range. Both MLF1 and SDC4 were not THCA-specific features.

\section{Potential function of LA16c-380H5.2, RP11- 203J24.8, MLF1 and SDC4}

To explore the functional implication of LA16c380H5.2 and RP11-203J24.8, both of which were uncharacterized previously in thyroid cells, in THCA tumorigenesis and development, we predicted lncRNA roles by using their targets. Among dysregulation pairs, KLF6 was the unique target of RP11-203J24.8, while the only two targets of LA16c-380H5.2 were, coincidentally, MLF1 and SDC4 (Figure 2B). The duplex structure of these two lncRNAs and their targets was predicted (Supplementary Figure 6A and 6B), and very low MFE (minimum free energy) indicated high accessibility for the sequence. The PCC between KLF6 and RP11203J24.8 was $0.71(\mathrm{P}=3.09 \mathrm{E}-10)$ in normal samples, but showed a sharp decrease $(\mathrm{PCC}=0.188, \mathrm{P}=2.15 \mathrm{E}-5)$ in the cancer group (Supplementary Figure 6C). The PCC
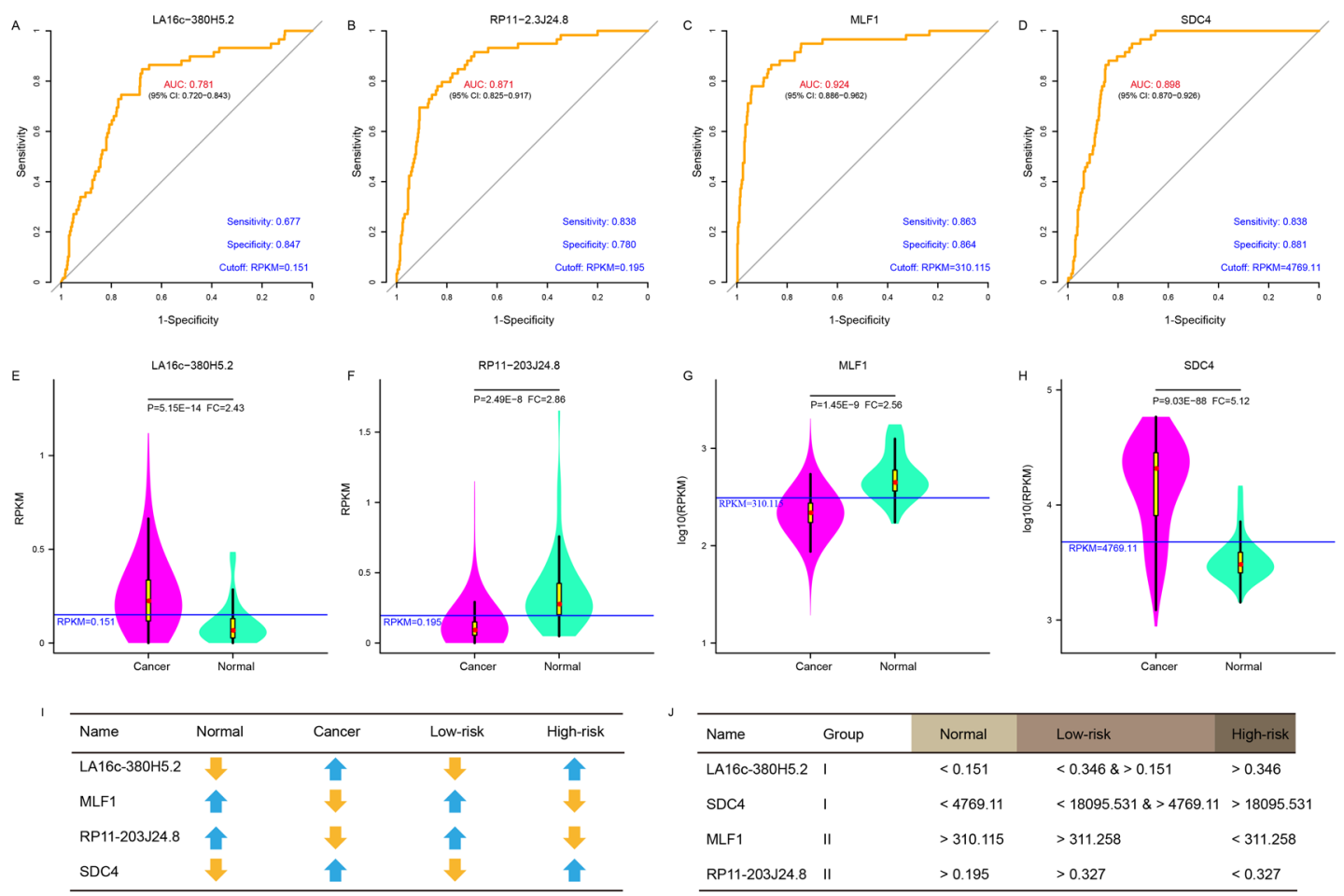

Figure 4: A panel of biomarker candidates with diagnostic values. ROC curve of LA16c-380H5.2 (A), RP11-203J24.8 (B), MLF1 (C) and SDC4 (D) showed high sensitivity and specificity to diagnose THCA patients from normal individuals. Comparison of expression levels in normal and cancer samples for LA16c-380H5.2 (E), RP11-203J24.8 (F), MLF1 (G) and SDC4 (H). (I) Comparison of expression change trends for four biomarkers in normal and cancer, high-risk and low-risk groups. (J) RPKM cutoff values of LA16c380H5.2, RP11-203J24.8, MLF1 and SDC4 to distinguish the normal, the low-risk or the high-risk groups. 
between MLF1 or SDC4 and LA16c-380H5.2 was 0.779 $(\mathrm{P}=3.62 \mathrm{E}-13)$ and $0.85(\mathrm{P}=1.77 \mathrm{E}-17)$, respectively, from normal samples and $0.058(\mathrm{P}=0.194)$ and 0.35 $(\mathrm{P}=5.87 \mathrm{E}-16)$ for the cancer group (Supplementary Figure $6 \mathrm{D}$ and $6 \mathrm{E}$ ); both PCC values fell considerably. These results illustrate that these three dysregulation pairs were affiliated with the Type I (Table 2), meaning that LA16c-380H5.2 and RP11-203J24.8 lost some targets in THCA, which may be partly because of the strict criteria of $|\mathrm{PCC}|>0.7$ and $\mathrm{P}<0.001$. Therefore, we focused on their targets in normal samples. A total of 147 PCGs were positively or negatively correlated with LA16c-380H5.2 (Supplementary Table 6), and 11 PCGs were positively or negatively correlated with RP11203J24.8 (Supplementary Table 7). The 147 PCGs were significantly enriched $(\mathrm{P}<0.05$ and Fold Enrichment $>2$ ) in $67 \mathrm{GO}$ (Gene Ontology) terms and $13 \mathrm{KEGG}$ (Kyoto encyclopedia of genes and genomes) pathways (Supplementary Table 8), including "negative regulation of cell proliferation" ( $\mathrm{P}=2.41 \mathrm{E}-4)$, "negative regulation of cell growth" $(\mathrm{P}=0.014)$, "negative regulation of cell cycle" $(\mathrm{P}=0.03)$, "negative regulation of apoptotic process" ( $\mathrm{P}=0.009)$, "activation of MAPK activity" ( $\mathrm{P}$ $=0.009)$, and "positive regulation of cell migration" $(\mathrm{P}=$ 0.01). Except for those GO functional terms that seemed conflicting in function, some important KEGG pathways that are involved in cancers were also enriched, such as "TNF signaling pathway" $(\mathrm{P}=0.003)$, "p53 signaling pathway" ( $\mathrm{P}=0.003)$, "MAPK signaling pathway" ( $\mathrm{P}=$ 0.028 ) which was reported by different studies on THCA $[2,4,17,28]$, and "PI3K-Akt signaling pathway" ( $\mathrm{P}=$ 0.04), showing that the IncRNA LA16c-380H5.2 may promote normal growth and development of thyroid cells. By detecting expression in 505 THCA and 59 normal tissues, we found significantly higher expression of LA16c-380H5.2 in patients (Figure 4E), suggesting that LA16c-380H5.2 may be considered as an oncogene in the thyroid and may be a potential novel therapeutic target for THCA patients. For targets of lncRNA RP11-203J24.8,
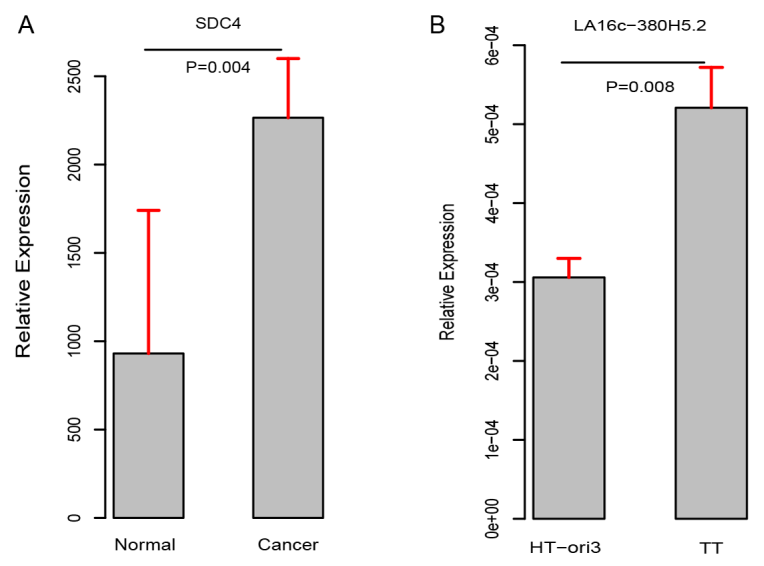

we found no KEGG pathways enriched, and only one enriched GO term: "intracellular signal transduction" $(\mathrm{P}=0.023)$; its target KLF6 was reported as a cancer driver gene [52] that also showed importance in thyroid normality maintenance.

SDC4 (syndecan 4) encodes a type I transmembrane heparan sulfate proteoglycan that is a main cell adhesion receptor involved in focal adhesion formation, and is required for cell migration [53, 54]. SDC4 has been reported as a cancer driver gene [52] and was up-regulated in our study $(\mathrm{FC}=5.12$, Figure $4 \mathrm{H})$, which was in accordance with two previous studies using microarrays [4, 25]; Griffith et al. reported a 3.32 FC [25], and Chung et al. detected a 3.90 FC [4]. These results were consistent with the high expression of SDC4 in other malignances (Figure 6F), indicating the oncogene nature of SDC4 in THCA development. MLF1 (myeloid leukemia factor 1) encodes an oncoprotein that plays a role in the phenotypic determination of hemopoietic cells [55]. Translocations between MLF1 and nucleophosmin have been associated with myelodysplastic syndrome (MDS) and acute myeloid leukemia (AML) [56]. Increased MLF1 as an oncogene expression has been correlated with a poor prognosis in AML and with malignant progression in $\operatorname{MDS}$ [57, 58]; however, it was significantly down-regulated in THCA (Figure 4G) and expressed at a lower level in the high risk group than in the low risk group (Figure 3H). MLF1's lowest expression was marvellously in AML among various cancers, and its expression level in THCA was obviously higher than in AML (Figure 6E). Just like SDC4, MLF1 also occupied a critical position in the network and had more interactions than the average gene (Figure 2D), which are common characteristics of cancer driver genes $[59,60]$. These results suggested that MLF1 may have an unknown novel function in THCA as a potential cancer driver gene and may maintain the normal cellular growth and development of thyroid, as well as suppressed roles in THCA tumorigenesis.
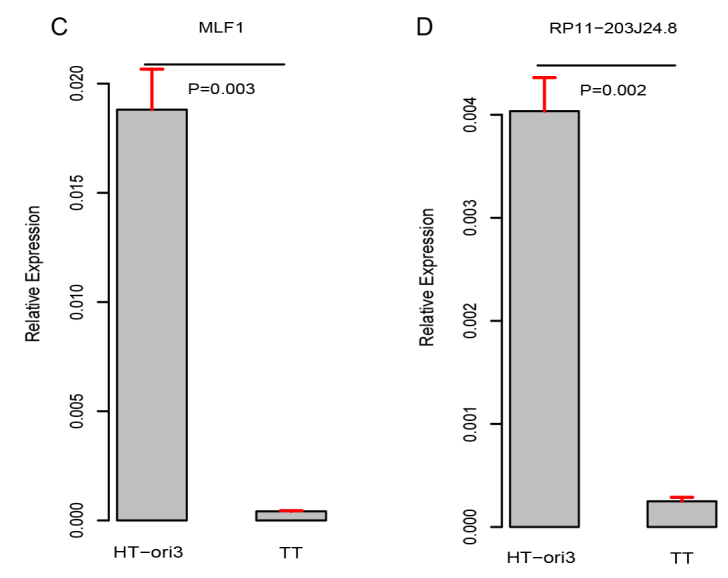

Figure 5: Validation of expression pattern of these four genes (A-D). 


\section{DISCUSSION}

Successful cancer treatment depends heavily on early detection [44]; however, few biomarkers are routinely used in clinics [61]. Although THCA is a relatively indolent cancer with low mortality [6], it frequently (30-90\% of patients) metastasizes in the lymph node [62]. These often predicts poor prognoses in THCA patients in the cervical region [63]. Persistent and recurrent disease rates remain significant [64]. Therefore, it is becoming increasingly important to find reliable and clinically applicable novel biomarkers for diagnosis or prediction.

In THCA, some molecular markers (such as SDC4, PAX8, TTF1, miR-181b and miR-221) have been evaluated to improve preoperative diagnostic or predictive accuracy in patients $[25,46,47,65,66]$. For example, Griffith et al. reported 39 differentially expressed genes (23 up-regulated and 16 down-regulated, $\mathrm{P}<0.05$ ) in 473 samples [25], and Chung et al. identified 79 differentially expressed genes (70 up-regulated and 9 down-regulated, FC $>3$ and FDR $<0.01)$ in 26 samples [4]. Among the up-regulated genes, seven genes (MET, TGFA, PROS1, PSD3, SDC4, TUSC3, and P4HA2) were detected in both reports. Only one gene was selected as a biomarker in our study, SDC4 $(\mathrm{P}=9.03 \mathrm{E}-88, \mathrm{FC}=5.12$, Figure $4 \mathrm{H})$, showing that SDC4 is robustly up-regulated in various THCA populations across different studies. While PAX8 and TTF1 might not be exclusive enough to be biomarkers based on our evaluation (Supplementary Figure 2C and 2D).

In addition to genetic factors, increasing evidence has suggested the implication of lncRNAs in the process of cancer occurrence and progression [67,68]. The IncRNA can significantly inhibit/promote proliferation, migration and apoptosis of a cell, and it is an attractive way to diagnose and cure cancers via the effective control of both cell growth and motility through lncRNAs [10, 69]. However, only a small amount of lncRNAs are known to be involved in THCA pathogenesis. For example, BANCR (BRAF-activated lncRNA) could increase cell proliferation in papillary thyroid cancer (PTC, PTC accounts for approximately $80 \%$ of all THCA in adults [70]), and its levels were significantly higher in PTC [18]. PTCSC3 (papillary thyroid carcinoma susceptibility candidate 3) is a tumor suppressor, and was downregulated in PTC $[2,19,71]$. NAMA (noncoding RNA associated with MAP kinase pathway and growth arrest) was found to be down-regulated in PTC and associated with arresting growth [17]. The expression of PVT1 (LINC00079), which contributes to tumorigenesis through recruiting EZH2 (polycomb enhancer of zeste homolog 2) and regulating TSHR (thyroid-stimulating hormone receptor), was significantly up-regulated in THCA [20]. All these reports show that lncRNA expression abnormality may be one reason of cancer pathogenesis
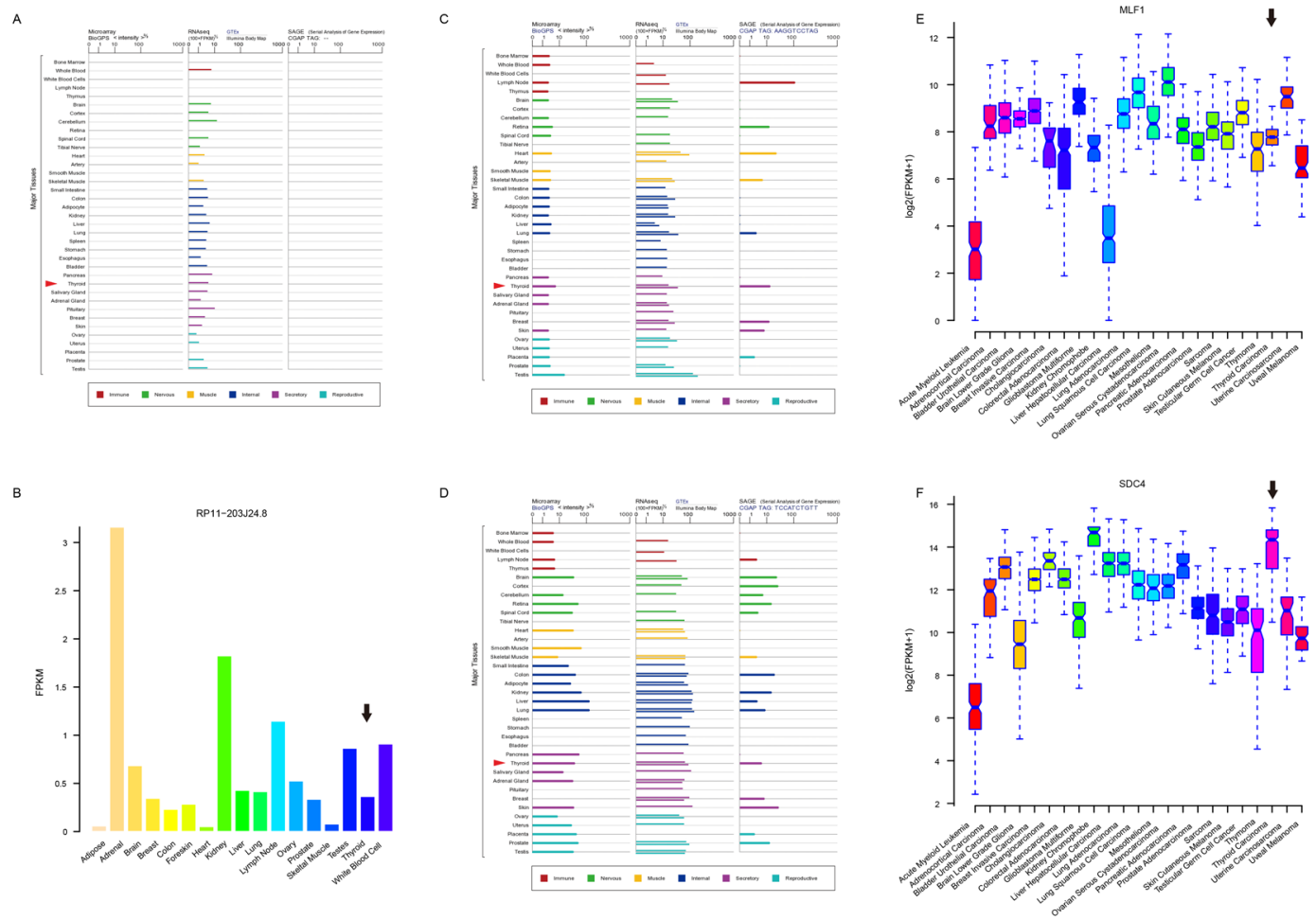

Figure 6: Expression profiles of four biomarker candidates in three public databases. Expression profiles of LA16c-380H5.2 (A), MLF1 (C) and SDC4 (D) in normal tissues from GeneCards database. Expression profiles of RP11-203J24.8 (B) in normal tissues from NONCODE database. Expression profiles of MLF1 (E) and SDC4 (F) in various cancers from cBioPortal database. 
and also suggested its potential as diagnostic biomarkers. In our study, the expression levels of PTCSC3 $(\mathrm{P}=9.21 \mathrm{E}-$ $17, \mathrm{FC}=0.41$, Supplementary Figure $2 \mathrm{E})$ and NAMA $(\mathrm{P}=$ 5.37E-16, FC $=0.29$, Supplementary Figure $2 \mathrm{~F}$ ) in THCA were concordant with those above studies, when compared with the normal samples. BANCR was not present in the GRCh37 reference. PVT1, with slight down-regulation in our study $(\mathrm{P}=0.03, \mathrm{FC}=0.746$, Supplementary Figure $2 \mathrm{G})$, was not defined as a differentially expressed lncRNA. Which may suggest that different environmental exposures may lead to various changes in diverse populations. This difference may also come from methods (namely, the qRT-PCR method and methods where cancer tissues are compared with adjacent normal tissues) and sample size (84 patients) in its study. Those above analyses about PCG and lncRNA were limited to a small number of samples, but the biomarkers we identified were more believable because of the advantages of a larger sample size and a more advanced platform of NGS. Recently, Qiuying $L i$ et al also reported four independent lncRNA biomarkers (RP11-536N17.1, RP11-508M8.1, AC026150.8 and CTD-2139B15.2), which were different from the four biomarkers identified in our study, associated with prognosis from the same TCGA-THCA dataset [72]. There are many difference between those two researches. Qiuying Li et al randomly divided THCA patients into two distinct sets of equal size: the 246-patient training dataset and the 246-patient testing dataset, they studied lncRNAs from the GENCODE Resource (version 19) and used TCGA RNA-sequencing data in the BAM file, and differentially expressed lncRNAs were identified using a paired student t-test, their prognostic biomarkers were focused on the survival and recurrence prediction. While we used the THCA patients as a whole, we focused on cancer PCGs and their regulating lncRNAs from the GRCH37 human genome assembly and used TCGA RNAsequencing data in the FASTQ format, and differentially expressed PCGs and lncRNAs were identified using Fisher's exact test or student's t test, biomarkers we identified were focused on their diagnostic values.

Furthermore, the number of DNA mutations is highly heterogeneous among various types of cancers [73]. Eduard Porta-Pardo et al. previously reported that THCA had only 11 missense mutations per sample (4420 mutations in 401 samples), which was the lowest in 23 types of cancer (5989 samples) from TCGA, while melanoma had the highest number, with 429 missense mutations per sample [52]. Since the relatively small number of DNA mutations in THCA patients may restrict the consideration of them as biomarkers, aberrantly expressed PCGs and lncRNAs of cancers could be a good alternative. Herein, we demonstrated the utility of a novel panel of diagnostic biomarker candidates including two PCGs (MLF1 and SDC4) and two lncRNAs (LA16c380H5.2 and RP11-203J24.8) from the dysregulation network based on expression signatures (Figure 4J).
Although the number of samples used for creating this panel was limited, the strict criteria used for selecting them provides a strong signature for biomarker validation. Fine needle aspiration, which is considered a gold standard for differential diagnosis, has a diagnostic sensitivity of 0.83 0.98 and specificity of $0.70-0.92$ in THCA [74, 75]. Here, we obtained a satisfying result with a sensitivity of 0.677 0.863 and specificity of $0.780-0.881$ (Figure 4A-4D). The improved ability to detect RNA, especially by in situ hybridization, RT-PCR, and sequencing techniques, will make RNA easily accessible to clinical applications and will improve the diagnosis or prognosis of THCA.

However, there are some limitations that should be acknowledged in our study. It should be emphasized that these four identified potential biomarkers were predicted by bioinformatic methods, and they were just validated in cell lines. Although substantial computational evidence for the diagnostic significance had been revealed, the underlying mechanisms of these four biomarkers in the development of THCA are still unclear. Both tumors and normal tissues are complex mixtures that include multiple types of cells, such as cancer cells, infiltrating lymphocytes and blood vessels, and variations in gene expression may thus simply reflect differences in cell composition [44]. Sample heterogeneity with respect to types of THCA, clinical activity and severity might impair the analysis. Random changes that exist in patients may be another confounding factor; for example, GAPDH is a house-keeping gene and is often used in in vivo disc research [76]; however, its expression levels changed markedly in both the THCA group and the normal group (Supplementary Figure 2H). The catalogue of cancer biomarkers is far from complete, and it is difficult to extend it by simply increasing the size of samples [77]. An alternative approach towards that goal is to integrate various types of biological knowledge to increase the statistical power of the analysis [52]. Moreover, because of extreme complexity and individual diversity, the current gene-centric paradigm in cancer biology may not be enough to explain the complex genotype-phenotype relationships [78-80]. It is expected that the information of DNA and epigenetic variation and protein-protein interaction profiles of cancer patients will be combined with our RNA-centric analysis.

\section{MATERIALS AND METHODS}

\section{Sequencing data analysis}

The results shown here are in whole based upon data generated by the TCGA Research Network: http:// cancergenome.nih.gov/. The downloaded RNA sequencing data used in this study are in FASTQ format, including 505 patients and 59 healthy individuals; those 564 sample IDs are provided in Supplementary Table 1. Bowtie software [81] with the default parameters was used to align RNA 
sequencing reads (48 nucleotides length) to the GRCH37 human genome assembly downloaded from the Ensembl database (http://asia.ensembl.org). These uniquely mapped reads in the genome were used to identify unambiguous transcription. Reads aligned to more than one locus were discarded. The expression level of transcripts was quantified by calculating the RPKM (reads per kilobase per million mapped) value. Genes were categorized as "protein coding" and "non-coding" based on an Ensembl annotation file in the GTF format. Among non-coding genes, rRNAs, tRNAs, miRNAs, snoRNAs and other known classes of RNAs were excluded, and lncRNAs were defined as all non-coding genes longer than 200 nucleotides and not belonging to other RNA categories. There were 18078 protein coding genes (PCGs) and 12727 IncRNA in the reference.

\section{Differential expression analysis of cancer and normal samples}

To identify the difference in PCG or lncRNA expression in cancer samples versus normal samples, we used the Wilcoxon rank sum test. For each feature (lncRNA or PCG), the read count was used as the input expression datum; we then calculated the frequency of expression in cancer and normal samples. If the ratio of the zero-value for the expression level (not detected, read count $=0$ ) was more than $30 \%$ in cancer or in normal samples, a twosided Fisher's exact test with an adjustment for multiple testing by the Benjamini-Hochberg method was used, with the thresholds of FDR $<0.01$ and $|\mathrm{FC}|$ (absolute value of the fold change) $>2$. Otherwise, Student's t test with the same adjustment was applied, with FDR $<0.05$ and $|\mathrm{FC}|$ $>2$. The FC was calculated by taking the higher mean RPKM value divided by the lower mean RPKM value in the THCA or normal samples. Unsupervised hierarchical clustering was done by $\mathrm{R}$ software (version 3.3.2, http:// www.r-project.org/).

Expression profiles of SDC4 and MLF1 in various cancers were queried in the cBioPortal (http:// www.cbioportal.org) [50]. LA16c-380H5.2, SDC4, and MLF1 expression values in major normal tissues were downloaded from the GeneCards (http://www.genecards. org) [49]. RP11-203J24.8 expression profiles in major normal tissues were retrieved from the NONCODE (http:// www.noncode.org) [51].

\section{Construction of the regulatory network}

Pearson's correlation coefficient (PCC) was calculated by in-house R- scripts, and was utilized to evaluate the co-expression relationship between lncRNA and PCG. Co-expressed pairs were defined with a cutoff of $|\mathrm{PCC}| \geq 0.7$ and $\mathrm{P}<0.001$. Network interactions were graphed using Cytoscape software (version 3.2.1) [82]. Two sub-modules were identified by CytoCluster
[83], with the HC-PIN clustering algorithm and other parameters as default, which is a plugin in Cytoscape.

\section{Data visualization}

The Kaplan-Meier survival curves of over-all survival was obtained using the Survival package [84]. The receiver operating characteristic (ROC) and the area under the ROC curves (AUC) values were obtained from the pROC package [85]. Unless otherwise specified, data were analyzed and visualized using $\mathrm{R}$ software (version 3.3.2).

\section{Duplex structure prediction}

The duplex structure was predicted by RNAplex and RNAduplex in the ViennaRNA package [86], using the DNA sequence of the PCG with 2000bp upstream and downstream and the RNA sequence of the lncRNA, which were retrieved from the GRCH37 human genome reference. The range of the predicted duplex structure in the two sequences is in the format "from, to : from, to". The minimum free energy (MFE) is in $\mathrm{kcal} / \mathrm{mol}$. The predicted structure is in the dot-bracket format with a "\&" separating the two sequences. A dot in the format represents an unpaired position, while a base pair $(i, j)$ is represented by a pair of matching parentheses at positions $\mathrm{i}$ and $\mathrm{j}$.

\section{Pathway analysis}

For enrichment analysis to explore their biological effects, PCGs were submitted to the DAVID (http://david. abcc.ncifcrf.gov/) [87]. The reporting of the enriched results was limited to Gene Ontology (GO) terms and Kyoto encyclopedia of genes and genomes (KEGG) pathway categories using the functional annotation clustering and functional annotation chart options. The GO terms and KEGG pathways with $\mathrm{P}$ values of $<0.05$ were considered as significantly enriched function annotations.

\section{Reagents and cell culture}

F12K medium (Life, Cat. 21127022), RPMI 1640 medium (Gibco, Cat. 11875093), DMEM(H) (Gibco, Cat. 11965092), NEAA (Invitrogen, Cat. 11140050), Glutamax (Invitrogen, Cat. 35050061), Sodium Pyruvate (Invitrogen, Cat. 11360070), Fetal bovine serum (Gibco, Cat. 10099141), penicillin/streptomycin solution (P/S, Cat. 0503), TRIzol ${ }^{\mathrm{TM}}$ Reagent (Invitrogen, Cat. 15596018), reverse transcription kit (Takara Bio Inc, Liaoning, China, Cat. RR036B $(A \times 4)$ ), SYBR Premix Ex Taq kit (Takara Bio Inc, Liaoning, China, Cat. RR420B).

The human thyroid medullary carcinoma cell line (TT), human papillary thyroid carcinoma cell lines (B-CPAP and BHT101) and human normal thyroid cell line (HT-ori3) were used in the current study. Those cell 
lines were purchased from Stem Cell Bank, Chinese Academy of Sciences (Shanghai, China). TT cells were cultured with $\mathrm{F} 12 \mathrm{~K}$ medium containing $10 \%$ (vol/vol) FBS and $1 \%$ (vol/vol) $\mathrm{P} / \mathrm{S}$ at $37^{\circ} \mathrm{C}$ with $5 \% \mathrm{CO}_{2}$. B-CPAP cells were cultured with RPMI 1640 medium containing $10 \%$ (vol/vol) FBS, 1\% (vol/vol) P/S, 1\% NEAA, 1\% Glutamax and $1 \%$ Sodium Pyruvate at $37^{\circ} \mathrm{C}$ with $5 \% \mathrm{CO}_{2}$. BHT101 cells were cultured with $\operatorname{DMEM}(\mathrm{H})$ medium containing $20 \%$ (vol/vol) FBS and $1 \%$ (vol/vol) P/S. HTori3 cells were cultured with 1640 medium containing $10 \%$ (vol/vol) FBS and 1\% (vol/vol) P/S.

\section{RNA isolation}

TT cells, B-CPAP cells, BHT101 cells, and HT-ori3 cells were cultured in the specific medium and harvested at $80 \%$ density. The cell pellets were dissolved in $1 \mathrm{ml}$ Trizol solution and isolated according to a stand RNA isolation procedure. The RNA concentration in each sample was quantified using a spectrophotometer at $260 \mathrm{~nm}$, the purity of RNA was assessed by measuring OD260/OD280 ratio (range 1.85-2.00).

\section{Real-time PCR}

Total RNA of different cell lines was extracted, and then the cDNA were synthesized with reverse transcription. Real-time PCR reactions were carried out in a final volume of 25 ul, using SYBR Premix ExTaq kit, $0.4 \mathrm{mM}$ of each primer, and $200 \mathrm{ng}$ of cDNA template. Each individual sample was run in triplicate wells. PCR amplification cycles were performed using $\mathrm{iQ}^{\mathrm{TM}} 5$ Multicolor Real-Time PCR Detection System (Bio-RAD). The reactions were initially denatured at $95^{\circ} \mathrm{C}$ for $3 \mathrm{~min}$, followed by 50 cycles of $95^{\circ} \mathrm{C}$ for $10 \mathrm{~s}, 55^{\circ} \mathrm{C}$ for $15 \mathrm{~s}, 72^{\circ} \mathrm{C}$ for $35 \mathrm{~s}$. The expression levels were calculated using the $2^{-\Delta \Delta C t}$ method and normalized to $\beta$-actin. The sequences of oligonucleotide primers were showed in Supplementary Table 9, primers were designed using NCBI primerblast (http://www.ncbi.nlm.nih.gov/tools/primer-blast/). Experimental data were presented as the mean $\pm \mathrm{SD}$. Statistical significance between the groups was calculated by a two-tailed Student's t test.

\section{Abbreviations}

AML: acute myeloid leukemia; AUC: area under the ROC curves; BANCR: BRAF-activated lncRNA; $\mathrm{EZH} 2$ : polycomb enhancer of zeste homolog 2; FC: fold change; FPKM: fragments per kilobase per millions; GO: Gene Ontology; KEGG: Kyoto encyclopedia of genes and genomes; lncRNA: long non-coding RNA; MDS: myelodysplastic syndrome; MFE: minimum free energy; MLF1: myeloid leukemia factor 1; NAMA: noncoding RNA associated with MAP kinase pathway and growth arrest; NGS: the next generation sequencing; PAX8: paired box 8; PCC: pearson correlation coefficient; PCG: protein coding gene; PTC: papillary thyroid cancer; PTCSC3: papillary thyroid carcinoma susceptibility candidate 3; ROC: receiver operating characteristic; RPKM: the reads per kilobase per million mapped; SDC4: syndecan 4; TCGA: The Cancer Genome Atlas; THCA: thyroid carcinoma; TSHR: thyroid-stimulating hormone receptor; TTF1: thyroid transcription factor 1 .

\section{Author contributions}

Conception and design: XIW and YfD; Development of methodology: XIW and $\mathrm{ZmC}$; Acquisition of data: YfD; Analysis and interpretation of data: XlW and $\mathrm{ZmC}$; Writing, review, and/or revision of the manuscript: XlW, ZmC and LsM; Reagent and experiment: QZ; Administrative, technical, or material support: LsM; Study supervision: ZmC and LsM. All authors read and approved the final manuscript.

\section{ACKNOWLEDGMENTS}

The authors are indebted to their colleagues (Dr. Zepeng Qu and Lan Yang) for their valuable discussions and comments to the manuscript. They acknowledge the TCGA Research Network.

\section{CONFLICTS OF INTEREST}

The authors declare no conflicts of interest.

\section{GRANT SUPPORT}

This work was supported by National Key R\&D Program of China (2017YFC1103704) Sanming Project of Medicine in Shenzhen (SZSM201412020), Fund for High Level Medical Discipline Construction of Shenzhen (2016031638), Shenzhen Foundation of Science and Technology (JCJY20160229204849975, GJHZ20170314171357556), Shenzhen Foundation of Health and Family Planning Commission (SZXJ2017021), the National Natural Science Foundation of China (31601063), the Science and Technology Planning Project of Guangdong Province, China (2016A020226025), and the Shenzhen Foundation of Science and Technology (JCYJ20160229204849975, GCZX2015043017281705).

\section{REFERENCES}

1. Xu B, Shao Q, Xie K, Zhang Y, Dong T, Xia Y, Tang W. The Long Non-Coding RNA ENST00000537266 and ENST00000426615 Influence Papillary Thyroid Cancer Cell Proliferation and Motility. Cellular Physiology and Biochemistry. 2016; 38:368-378.

2. Lan X, Zhang H, Wang Z, Dong W, Sun W, Shao L, Zhang T, Zhang D. Genome-wide analysis of long noncoding RNA 
expression profile in papillary thyroid carcinoma. Gene. 2015; 569:109-117.

3. Williams WW, Taheri D, Tolkoff-Rubin N, Colvin RB. Clinical role of the renal transplant biopsy. Nature reviews Nephrology. 2012; 8:110-121.

4. Chung KW, Kim SW, Kim SW. Gene expression profiling of papillary thyroid carcinomas in Korean patients by oligonucleotide microarrays. Journal of the Korean Surgical Society. 2012; 82:271-280.

5. Yoon JH, Kim EK, Kwak JY, Moon HJ. Effectiveness and Limitations of Core Needle Biopsy in the Diagnosis of Thyroid Nodules: Review of Current Literature. Journal of Pathology and Translational Medicine. 2015; 49:230-235.

6. LiVolsi VA. Papillary thyroid carcinoma: an update. Modern Pathology. 2011; 24:S1-S9.

7. Naesens M, Sarwal MM. Molecular diagnostics in transplantation. Nature reviews Nephrology. 2010; 6:614-628.

8. Mercer TR, Dinger ME, Mattick JS. Long non-coding RNAs: insights into functions. Nat Rev Genet. 2009; 10:155-159.

9. Prensner JR, Chinnaiyan AM. The emergence of 1ncRNAs in cancer biology. Cancer Discov. 2011; 1:391-407.

10. Iyer MK, Niknafs YS, Malik R, Singhal U, Sahu A, Hosono Y, Barrette TR, Prensner JR, Evans JR, Zhao S. The landscape of long noncoding RNAs in the human transcriptome. Nature genetics. 2015; 47:199-208.

11. Ulitsky I, Bartel David P. lincRNAs: Genomics, Evolution, and Mechanisms. Cell. 2013; 154:26-46.

12. Boon RA, Jae N, Holdt L, Dimmeler S. Long Noncoding RNAs: From Clinical Genetics to Therapeutic Targets? Journal of the American College of Cardiology. 2016; 67:1214-1226.

13. Cheetham SW, Gruhl F, Mattick JS, Dinger ME. Long noncoding RNAs and the genetics of cancer. Br J Cancer. 2013; 108:2419-2425.

14. Zhou M, Zhao H, Wang Z, Liang C, Yang L, Shi H, Yang H, Sun J. Identification and validation of potential prognostic lncRNA biomarkers for predicting survival in patients with multiple myeloma. Journal of Experimental \& Clinical Cancer Research. 2015; 34:102.

15. Zhang K, Luo Z, Zhang Y, Zhang L, Wu L, Liu L, Yang J, Song X, Liu J. Circulating lncRNA H19 in plasma as a novel biomarker for breast cancer. Cancer Biomarkers. 2016; 17:187-194.

16. Yang Y, Shao Y, Zhu M, Li Q, Yang F, Lu X, Xu C, Xiao B, Sun Y, Guo J. Using gastric juice lncRNA-ABHD11-AS1 as a novel type of biomarker in the screening of gastric cancer. Tumor Biology. 2016; 37:1-6.

17. Yoon H, He H, Nagy R, Davuluri R, Suster S, Schoenberg $\mathrm{D}$, Pellegata N, Chapelle Ade L. Identification of a novel noncoding RNA gene, NAMA, that is downregulated in papillary thyroid carcinoma with BRAF mutation and associated with growth arrest. International Journal of Cancer. 2007; 121:767-775.
18. Wang Y, Guo Q, Zhao Y, Chen J, Wang S, Jun H, Sun Y. BRAF-activated long non-coding RNA contributes to cell proliferation and activates autophagy in papillary thyroid carcinoma. Oncology Letters. 2014; 8:1947-1952.

19. Fan M, Li X, Jiang W, Huang Y, Li J, Wang Z. A long non-coding RNA, PTCSC3, as a tumor suppressor and a target of miRNAs in thyroid cancer cells. Experimental \& Therapeutic Medicine. 2013; 5:1143-1146.

20. Zhou Q, Chen J, Feng J, Wang J. Long noncoding RNA PVT1 modulates thyroid cancer cell proliferation by recruiting EZH2 and regulating thyroid-stimulating hormone receptor (TSHR). Tumor Biology. 2016; 37:3105-3113.

21. Zhang R, Hardin H, Chen J, Guo Z, Lloyd RV. Non-Coding RNAs in Thyroid Cancer. Endocrine Pathology. 2016; 27:12-20.

22. The Cancer Genome Atlas Research N, Weinstein JN, Collisson EA, Mills GB, Shaw KRM, Ozenberger BA, Ellrott K, Shmulevich I, Sander C, Stuart JM. The Cancer Genome Atlas Pan-Cancer analysis project. Nat Genet. 2013; 45:1113-1120.

23. Derrien T, Johnson R, Bussotti G, Tanzer A, Djebali S, Tilgner H, Guernec G, Martin D, Merkel A, Knowles DG. The GENCODE v7 catalog of human long noncoding RNAs: analysis of their gene structure, evolution, and expression. Genome Research. 2012; 22:1775-1789.

24. Cabili MN, Trapnell C, Goff L, Koziol M, Tazon-Vega B, Regev A, Rinn JL. Integrative annotation of human large intergenic noncoding RNAs reveals global properties and specific subclasses. Genes \& Development. 2011; 25:1915-1927.

25. Griffith OL, Melck A, Jones SJM, Wiseman SM. MetaAnalysis and Meta-Review of Thyroid Cancer Gene Expression Profiling Studies Identifies Important Diagnostic Biomarkers. Journal of Clinical Oncology. 2006; 24:5043-5051.

26. Raman P, Koenig RJ. Pax-8-PPAR- $\gamma$ fusion protein in thyroid carcinoma. Nat Rev Endocrinol. 2014; 10:616-623.

27. Omur O, Baran Y. An update on molecular biology of thyroid cancers. Critical Reviews in Oncology / Hematology. 2014; 90:233-252.

28. Carpi A, Mechanick JI, Saussez S, Nicolini A. Thyroid tumor marker genomics and proteomics: Diagnostic and clinical implications. Journal of Cellular Physiology. 2010; 224:612-619.

29. Uhlén M, Fagerberg L, Hallström BM, Lindskog C, Oksvold P, Mardinoglu A, Sivertsson A, Kampf C, Sjöstedt E, Asplund A, Olsson I, Edlund K, Lundberg E, et al. Proteomics. Tissue-based map of the human proteome. Science. 2015; 347:394.

30. Gibb EA, Brown CJ, Lam WL. The functional role of long non-coding RNA in human carcinomas. Molecular Cancer. 2011; 10:38-38.

31. Batista PJ, Chang HY. Long noncoding RNAs: cellular address codes in development and disease. Cell. 2013; 152:1298-1307. 
32. Yu G, Yao W, Gumireddy K, Li A, Wang J, Xiao W, Chen K, Xiao H, Li H, Tang K, Ye Z, Huang Q, Xu H. Pseudogene PTENP1 functions as a competing endogenous RNA to suppress clear-cell renal cell carcinoma progression. Mol Cancer Ther. 2014; 13:3086-3097.

33. Sun M, Kraus WL. From Discovery to Function: The Expanding Roles of Long Non-Coding RNAs in Physiology and Disease. Endocr Rev. 2015; 36:er00009999.

34. Li Z, Rana TM. Decoding the noncoding: prospective of lncRNA-mediated innate immune regulation. RNA biology. 2014; 11:979-985.

35. Jiang Q, Wang J, Wu X, Ma R, Zhang T, Jin S, Han Z, Tan R, Peng J, Liu G, Li Y, Wang Y. LncRNA2Target: a database for differentially expressed genes after lncRNA knockdown or overexpression. Nucleic Acids Res. 2015; 43:D193-D196.

36. Chen L, Xu L, Wang G. Regulation of MET-mediated proliferation of thyroid carcinoma cells by miR-449b. Tumor Biology. 2015; 36:8653-8660.

37. Bentzien F, Zuzow M, Heald N, Gibson A, Shi Y, Goon L, Yu P, Engst S, Zhang W, Huang D, Zhao L, Vysotskaia $\mathrm{V}$, Chu F, et al. In Vitro and In Vivo Activity of Cabozantinib (XL184), an Inhibitor of RET, MET, and VEGFR2, in a Model of Medullary Thyroid Cancer. Thyroid. 2013; 23:1569-1577.

38. Kondo T, Zheng L, Liu W, Kurebayashi J, Asa SL, Ezzat S. Epigenetically Controlled Fibroblast Growth Factor Receptor 2 Signaling Imposes on the RAS/BRAF/ Mitogen-Activated Protein Kinase Pathway to Modulate Thyroid Cancer Progression. Cancer Research. 2007; 67:5461-5470.

39. St Bernard R, Zheng L, Liu W, Winer D, Asa SL, Ezzat S. Fibroblast Growth Factor Receptors as Molecular Targets in Thyroid Carcinoma. Endocrinology. 2005; 146:1145-1153.

40. Wuchty S. Scale-free behavior in protein domain networks. Mol Biol Evol. 2001; 18:1694-1702.

41. Featherstone DE, Broadie K. Wrestling with pleiotropy: Genomic and topological analysis of the yeast gene expression network. BioEssays. 2002; 24:267-274.

42. Ravasz E, Somera AL, Mongru DA, Oltvai ZN, Barabási AL. Hierarchical Organization of Modularity in Metabolic Networks. Science. 2002; 297:1551-1555.

43. He H, Jazdzewski K, Li W, Liyanarachchi S, Nagy R, Volinia S, Calin GA, Liu CG, Franssila K, Suster $\mathrm{S}$, Kloos RT, Croce CM, de la Chapelle A. The role of microRNA genes in papillary thyroid carcinoma. Proceedings of the National Academy of Sciences of the United States of America. 2005; 102:19075-19080.

44. Kaczkowski B, Tanaka Y, Kawaji H, Sandelin A, Andersson R, Itoh M, Lassmann T, Hayashizaki Y, Carninci P, Forrest ARR, FANTOM5 Consortium. Transcriptome Analysis of Recurrently Deregulated Genes across Multiple Cancers Identifies New Pan-Cancer Biomarkers. Cancer research. 2016; 76:216-226.
45. Cabanski CR, White NM, Dang HX, Silva-Fisher JM, Rauck CE, Cicka D, Maher CA. Pan-cancer transcriptome analysis reveals long noncoding RNAs with conserved function. RNA biology. 2015; 12:628-642.

46. Suzuki A, Hirokawa M, Takada N, Higuchi M, Yamao N, Kuma S, Daa T, Miyauchi A. Diagnostic significance of PAX8 in thyroid squamous cell carcinoma. Endocrine Journal. 2015; 62:991-995.

47. Gao Y, Chen F, Niu S, Lin S, Li S. Replication and MetaAnalysis of Common Gene Mutations in TTF1 and TTF2 with Papillary Thyroid Cancer. Medicine. 2015; 94:e1246.

48. Segev DL, Clark DP, Zeiger MA, Umbricht C. Beyond the Suspicious Thyroid Fine Needle Aspirate. Acta Cytologica. 2003; 47:709-722.

49. Belinky F, Bahir I, Stelzer G, Zimmerman S, Rosen N, Nativ N, Dalah I, Iny Stein T, Rappaport N, Mituyama T, Safran M, Lancet D. Non-redundant compendium of human ncRNA genes in GeneCards. Bioinformatics. 2013; 29:255-261.

50. Cerami E, Gao J, Dogrusoz U, Gross BE, Sumer SO, Aksoy BA, Jacobsen A, Byrne CJ, Heuer ML, Larsson E, Antipin Y, Reva B, Goldberg AP, et al. The cBio cancer genomics portal: an open platform for exploring multidimensional cancer genomics data. Cancer Discov. 2012; 2:401-404.

51. Zhao Y, Li H, Fang S, Kang Y, wu W, Hao Y, Li Z, Bu D, Sun N, Zhang MQ, Chen R. NONCODE 2016: an informative and valuable data source of long non-coding RNAs. Nucleic Acids Res. 2016; 44:D203-D208.

52. Porta-Pardo E, Garcia-Alonso L, Hrabe T, Dopazo J, Godzik A. A Pan-Cancer Catalogue of Cancer Driver Protein Interaction Interfaces. PLoS computational biology. 2015; 11:e1004518.

53. Carvallo L, Munoz R, Bustos F, Escobedo N, Carrasco H, Olivares G, Larrain J. Non-canonical Wnt signaling induces ubiquitination and degradation of Syndecan4. J Biol Chem. 2010; 285:29546-29555.

54. Wu H, Zhou Q, Xie J, Li GN, Chen QH, Kang LN, Xu B. Syndecan-4 shedding is involved in the oxidative stress and inflammatory responses in left atrial tissue with valvular atrial fibrillation. International Journal of Clinical and Experimental Pathology. 2015; 8:6387-6396.

55. Molzan M, Ottmann C. Subcellular localization of fulllength human myeloid leukemia factor 1 (MLF1) is independent of 14-3-3 proteins. Cellular and Molecular Biology Letters. 2013; 18:137-148.

56. Dumézy F, Renneville A, Mayeur-Rousse C, Nibourel O, Labis E, Preudhomme C. Acute myeloid leukemia with translocation $\mathrm{t}(3 ; 5)$ : new molecular insights. Haematologica. 2013; 98:e52-e54.

57. Matsumoto N, Yoneda-Kato N, Iguchi T, Kishimoto Y, Kyo T, Sawada H, Tatsumi E, Fukuhara S. Elevated MLF1 expression correlates with malignant progression from myelodysplastic syndrome. Leukemia. 2000; 14:1757-1765. 
58. Bras S, Martin-Lannerée S, Gobert V, Augé B, Breig O, Sanial M, Yamaguchi M, Haenlin M, Plessis A, Waltzer L. Myeloid leukemia factor is a conserved regulator of RUNX transcription factor activity involved in hematopoiesis. Proceedings of the National Academy of Sciences of the United States of America. 2012; 109:4986-4991.

59. Futreal PA, Coin L, Marshall M, Down T, Hubbard T, Wooster R, Rahman N, Stratton MR. A census of human cancer genes. Nature reviews Cancer. 2004; 4:177-183.

60. Jonsson PF, Bates PA. Global topological features of cancer proteins in the human interactome. Bioinformatics. 2006; 22:2291-2297.

61. Felder M, Kapur A, Gonzalez-Bosquet J, Horibata S, Heintz J, Albrecht R, Fass L, Kaur J, Hu K, Shojaei H, Whelan RJ, Patankar MS. MUC16 (CA125): tumor biomarker to cancer therapy, a work in progress. Molecular Cancer. 2014; 13:129.

62. Caron NR, Clark OH. Papillary thyroid cancer: Surgical management of lymph node metastases. Current Treatment Options in Oncology. 2005; 6:311-322.

63. Lundgren CI, Hall P, Dickman PW, Zedenius J. Clinically significant prognostic factors for differentiated thyroid carcinoma. Cancer. 2006; 106:524-531.

64. Davies L, Welch HG. Thyroid cancer survival in the United States: observational data from 1973 to 2005. Archives of otolaryngology--head \& neck surgery. 2010; 136:440-444.

65. Mazeh H, Mizrahi I, Halle D, Ilyayev N, Stojadinovic A, Trink B, Mitrani-Rosenbaum S, Roistacher M, Ariel I, Eid A, Freund HR, Nissan A. Development of a MicroRNABased Molecular Assay for the Detection of Papillary Thyroid Carcinoma in Aspiration Biopsy Samples. Thyroid. 2011; 21:111-118.

66. Pallante P, Visone R, Ferracin M, Ferraro A, Berlingieri MT, Troncone G, Chiappetta G, Liu CG, Santoro M, Negrini M, Croce CM, Fusco A. MicroRNA deregulation in human thyroid papillary carcinomas. Endocrine-related cancer. 2006; 13:497-508.

67. Tong YS, Wang XW, Zhou XL, Liu ZH, Yang TX, Shi WH, Xie HW, Lv J, Wu QQ, Cao XF. Identification of the long non-coding RNA POU3F3 in plasma as a novel biomarker for diagnosis of esophageal squamous cell carcinoma. Molecular Cancer. 2015; 14:3-3.

68. Feinberg AP, Ohlsson R, Henikoff S. The epigenetic progenitor origin of human cancer. Nat Rev Genet. 2006; 7:21-33.

69. Tripathi V, Shen Z, Chakraborty A, Giri S, Freier SM, Wu X, Zhang Y, Gorospe M, Prasanth SG, Lal A, Prasanth KV. Long noncoding RNA MALAT1 controls cell cycle progression by regulating the expression of oncogenic transcription factor B-MYB. PLoS Genet. 2013; 9:e1003368.

70. La Vecchia C, Malvezzi M, Bosetti C, Garavello W, Bertuccio P, Levi F, Negri E. Thyroid cancer mortality and incidence: A global overview. International Journal of Cancer. 2015; 136:2187-2195.

71. Jendrzejewski J, He H, Radomska HS, Li W, Tomsic J, Liyanarachchi S, Davuluri RV, Nagy R, de la Chapelle A. The polymorphism rs944289 predisposes to papillary thyroid carcinoma through a large intergenic noncoding RNA gene of tumor suppressor type. Proceedings of the National Academy of Sciences of the United States of America. 2012; 109:8646-8651.

72. Li Q, Li H, Zhang L, Zhang C, Yan W, Wang C. Identification of novel long non-coding RNA biomarkers for prognosis prediction of papillary thyroid cancer. Oncotarget. 2017; 8:46136-46144. http://doi.org/10.18632/oncotarget.17556.

73. Lawrence MS, Stojanov P, Polak P, Kryukov GV, Cibulskis K, Sivachenko A, Carter SL, Stewart C, Mermel CH, Roberts SA, Kiezun A, Hammerman PS, McKenna A, et al. Mutational heterogeneity in cancer and the search for new cancer-associated genes. Nature. 2013; 499:214-218.

74. Cooper DS, Doherty GM, Haugen BR, Kloos RT, Lee SL, Mandel SJ, Mazzaferri EL, McIver B, Pacini F, Schlumberger M, Sherman SI, Steward DL, Tuttle RM. Revised American Thyroid Association Management Guidelines for Patients with Thyroid Nodules and Differentiated Thyroid Cancer. Thyroid. 2009; 19:1167-1214.

75. Cibas ES, Ali SZ. The Bethesda System for Reporting Thyroid Cytopathology. Thyroid. 2009; 19:1159-1165.

76. Yurube T, Takada T, Hirata H, Kakutani K, Maeno K, Zhang Z, Yamamoto J, Doita M, Kurosaka M, Nishida K. Modified house-keeping gene expression in a rat tail compression loading-induced disc degeneration model. Journal of Orthopaedic Research. 2011; 29:1284-1290.

77. Ge X, Chen Y, Liao X, Liu D, Li F, Ruan H, Jia W. Overexpression of long noncoding RNA PCAT-1 is a novel biomarker of poor prognosis in patients with colorectal cancer. Medical Oncology. 2013; 30:588-588.

78. Porta Pardo E, Godzik A. Analysis of individual protein regions provides novel insights on cancer pharmacogenomics. PLoS computational biology. 2015; 11:e1004024.

79. Sahni N, Yi S, Zhong Q, Jailkhani N, Charloteaux B, Cusick ME, Vidal M. Edgotype: the link between genotype and phenotype. Current opinion in genetics \& development. 2013; 23:649-657.

80. Sahni N, Yi S, Taipale M, Fuxman Bass JI, CoulombeHuntington J, Yang F, Peng J, Weile J, Karras GI, Wang Y, Kovács IA, Kamburov A, Krykbaeva I, et al. Widespread Macromolecular Interaction Perturbations in Human Genetic Disorders. Cell. 2015; 161:647-660.

81. Langmead B, Trapnell C, Pop M, Salzberg SL. Ultrafast and memory-efficient alignment of short DNA sequences to the human genome. Genome Biol. 2009; 10:R25. 
82. Cline MS, Smoot M, Cerami E, Kuchinsky A, Landys N, Workman C, Christmas R, Avila-Campilo I, Creech M, Gross B, Hanspers K, Isserlin R, Kelley R, et al. Integration of biological networks and gene expression data using Cytoscape. Nat Protoc. 2007; 2:2366-2382.

83. Tang Y, Min L. A Cytoscape plugin for visualization and clustering analysis of protein interaction networks. Chinese Journal of Bioinformatics. 2014; 12:38-45.

84. Therneau TM, Grambsch PM. Modeling Survival Data: Extending the Cox Model. Technometrics. 2002; 44:85-86.
85. Robin X, Turck N, Hainard A, Tiberti N, Lisacek F, Sanchez JC, Müller M. pROC: an open-source package for R and S+ to analyze and compare ROC curves. Bmc Bioinformatics. 2011; 12:77-77.

86. Lorenz R, Bernhart SH, Höner zu Siederdissen C, Tafer H, Flamm C, Stadler PF, Hofacker IL. ViennaRNA Package 2.0. Algorithms for Molecular Biology. 2011; 6:26.

87. Huang W, Sherman BT, Lempicki RA. Systematic and integrative analysis of large gene lists using DAVID bioinformatics resources. Nat Protoc. 2009; 4:44-57. 